\title{
On the mechanism of detonation initiations
}

\author{
Z. Jiang ${ }^{1}$, H. Teng ${ }^{1}$, D. Zhang $^{1}$, S.V. Khomik ${ }^{2}$, and S.P. Medvedev ${ }^{2}$ \\ 1 LHD of Chinese Academy of Sciences, Institute of Mechanics, Beijing 100080, China \\ 2 Institute of Chemical Physics, Russia Academy of Sciences, 119991 Moscow, Russia
}

Summary. Two essential issues in detonation initiations were discussed in this paper, that is, hotspots and reaction zone acceleration. By defining the reaction zone acceleration, the hotspot and the reaction zone acceleration are identified as the primary mechanisms underlying the deflagration to detonation transition. The dominant physical process for two mechanisms is the interaction between gasdynamic non-linearity and chemical reactions that are very sensitive to temperature gradient. In the process, spontaneous waves are generated in the reaction zone, propagate with the local sound speed, decelerate suddenly in the front of the reaction zone because of the sharp temperature gradient, and develop into a pressure pulse acting to elevate the gas temperature that will further induce more intensive chemical reactions. Such a positive feedback loop supports detonation initiation and propagation.

\section{Introduction}

There are two modes of detonation initiations as classified by Lee [1]: a slow mode where the detonation is developed via an accelerating flame front and a fast mode where the detonation is driven out "instantaneously" by a strong blast that is created by a sufficiently powerful igniter. The slow mode is usually referred to as the self-initiation via the deflagration to detonation transition (short for, DDT). The fast mode is referred to as a direct initiation. However, even when the ignition energy is substantially high above the critical value for the direct initiation, the blast is always observed to decay first below the C-J value, and then, re-accelerates itself back to the C-J value at large radius [2]. This excursion is now known as the quasi-steady period before the final onset of detonation, and also considered to depend on DDT. So, it is believed that exploring DDT is not only important for the self-initiation, but also for the direct initiation.

DDT is a primary combustion phenomenon and has been called one of the major unsolved problems in combustion theory [3] for years because the mechanisms underlying DDT in gaseous energetic mixtures are still not well understood. many experiments on DDT were carried out in the past several decades and the pioneer work showed that DDT is an extremely complex process involving deflagrations, shock interactions, shear layers, turbulence, chemical reactions, flow instability, and all of their interactions with each other. Two issues had been reported to be important for DDT: hotspots and flame acceleration. The hotspots seem to be well understood than the flame acceleration because more complex processes are included in it. To develop an universal theory for describing the mechanisms underlying detonation initiation, more elementary phenomena need to be defined, which must be simple and not involved with each other.

This paper is dedicated to exploring primary mechanisms in detonation initiations by examining two essential issues, i.e. hotspot and reaction zone acceleration. Numerical simulations are carried out by solving two-dimensional multi-component Navier-Stokes 
equations implemented with an elementary chemical reaction model. Numerical results of two test cases are reported and discussed in detail.

\section{Problem specifications}

Figure 1(a) shows the sketch of toroidal shock focusing of the first case and its geometric domain consists of a shock tube and solid cylinder, which are co-axially installed with a given scale ratio of $d: D$. The whole domain is filled with the reactive gas of $2 \mathrm{H}_{2}+\mathrm{O}_{2}+4 \mathrm{~N}_{2}$ at the $1.0 \mathrm{~atm}$ pressure and the $300 \mathrm{~K}$ temperature. The incident shock wave is initially posited near the left boundary, and diffracts along the end of the solid cylinder, and then implodes toward the axis of symmetry. The diffracting shock wave will decay first, but get stronger and stronger as its radius decreases. The converging process deposits some energy around the focal point and provides hotspots with the required thermodynamic environment. The energy thus deposited can be adjusted by changing either the incident Mach number or the domain configuration scale, so that it can be convenient to generate a hotspot and trigger a detonation bubble at a expected location.
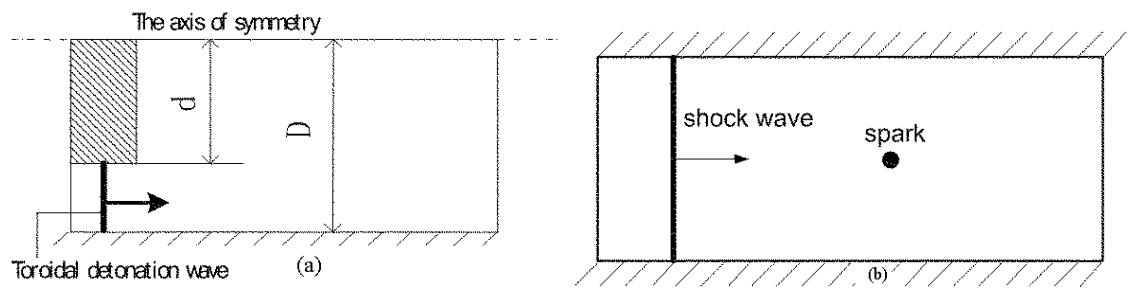

Fig. 1. Schematic of toroidal shock focusing and shock/spark-induced-deflagration interaction

The computational domain for the second test case is shown in Fig.1(b). This is the incident shock/spark-induced-deflagration interaction in a two-dimensional channel and was examined in many papers, for example, in Oran's work [3]. The deflagration front is generated from an electric spark and interacts with the coming incident shock. The detonation initiation will be induced from reaction zone instability.

\section{The role of hotspots in detonation initiations}

The "hotspot" has been recognized to be universal to all modes of detonation initiations since it was reported as "explosion in the explosion" by Oppenheim [4]. In his paper, a sequence of typical schlieren photographs demonstrated the hotspot was generated from a turbulent flame brush and developed into a detonation bubble. The experiments following his work indicated that the hotspot can originate either at flame fronts, boundary layers, disturbed contact surfaces and locations at which shock waves merged, and its appearance may vary from event to event. The exact physical nature of the process prior and subsequent to the hotspot explosion that leads to a detonation establishment has attracted many researchers for decades, but still remain to be not well understood. 

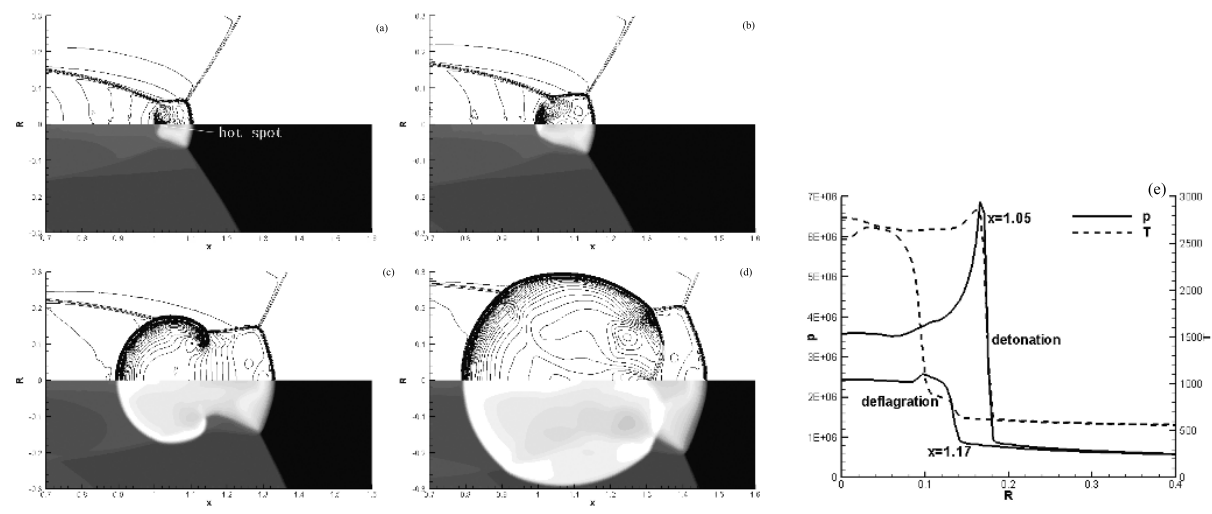

Fig. 2. Sequential pressure and temperature distributions during hotspot development with the pressure and the temperature profiles across detonation bubble at a given moment

Setting the incident Mach number to be 2.70 and 2.72 for the first case, two numerical experiments were carried out. Numerical results showed that the physical phenomena occurring in two tests are dramatically different with each other. For $M_{i}=2.70$, the shock reflection pattern is the same to the shock wave focusing in non-reactive gases and no significant chemical reactions are observable. For $M_{i}=2.72$, the results in the region around the focal point at four instants are plotted in Fig. 2 and showed that a hotspot was generated and developed in a detonation bubble later. At the beginning of shock focusing as shown in Fig.2(a), there is a hotspot occurring on the axis of symmetry. Inside the hotspot, the explosion-like chemical reactions generate intensive spontaneous waves as shown in Fig.2(b). These waves spread successively behind the reaction zone, but are accumulated in the front of the reaction zone because of sharp temperature gradient, and elevate gas thermo-states that can speed up chemical reactions there. A feedback loop develops quickly, and a complex of a shock and reaction zone appears, and looks like a mushroom referred as to detonation bubble, as shown in Fig. 2(c). The detonation bubble spreads quickly, and engulfs the reaction zone, as shown in Fig.2(d). Finally a closed detonation bubble generates and becomes self-sustained later.

Figure 2(e) shows the pressure and temperature profiles along two lines of $r=1.05$ and 1.17 at the same time instant of Fig.2(c). The profiles along the line of $r=1.17$ that passes the downstream part (right) of the mushroom-shaped reaction zone show that the shock wave and the reaction zone are decoupled. The post-shock temperature is about $800 \mathrm{~K}$, and reaches to $2700 \mathrm{~K}$ in the following reaction zone where a pressure drops slightly. Hence, the combustion in the downstream part is a typical deflagration. Examining the profiles along the line of $r=1.05$ that passes through the upstream part (left) of the mushroom-shaped reaction zone, one can see that the shock and the reaction zone couple tightly and the post-shock parameters reach to the C-J values. This typical profile indicates that the detonation bubble had developed.

To further investigate into detonation bubble developing, a time sequence of pressure, temperature, and $\mathrm{OH}$ and $\mathrm{H}_{2} \mathrm{O}$ profiles along the axis of symmetry at five instants are shown in Fig. 3. The time interval between the first and the last profile is about $0.53 \mathrm{mi}-$ croseconds, and the presented flow field is limited around the focal point. Examining the 

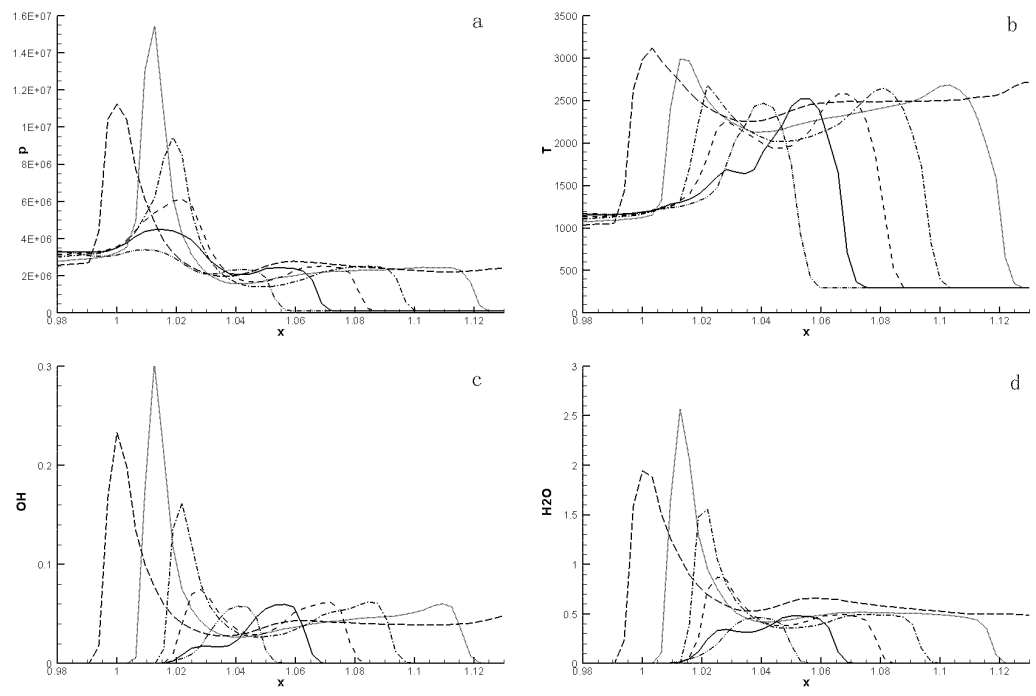

Fig. 3. Sequential profiles of pressure, temperature, $\mathrm{OH}$ and $\mathrm{H}_{2} \mathrm{O}$ during hotspot explosion

first profile, one can see that the peak temperature is about $2500 \mathrm{~K}$, and the corresponding pressure, $\mathrm{OH}$ and $\mathrm{H}_{2} \mathrm{O}$ are much low, therefore, the combustion at the moment is still in deflagration stage. The reaction zone travels both upstream and downstream. The downstream-travelling zone remains to be deflagration, but in the upstream-travelling zone, the post-shock parameters arise higher and higher with time evolution. Finally, a hotspot develops, as observed in Fig.3(a). The highest peak pressure appearing in front of the reaction zone at the fifth profile indicated with a solid line is about $15.5 \mathrm{MPa}$, the corresponding temperature is around $3000 \mathrm{~K}$, and $\mathrm{OH}$ and $\mathrm{H}_{2} \mathrm{O}$ also reach to their maximum values, as shown in Fig.3(b)-3(d). This implies that intensive chemical reactions result in a hotspot explosion from which a detonation bubble forms. Shortly after the instant, the leading shock wave decays as it propagates up-stream, and the temperature reaches and stays around $3000 \mathrm{~K}$ for a longer time, as shown at the sixth profile in Fig.3(b). This demonstrated that the detonation is overdriven at the beginning of detonation bubble developing and decays to the C-J values later. So, it is understood that the hotspot-induced detonation initiation behaves itself like the direct initiation.

\section{Reaction zone acceleration}

The flame acceleration had been reported and its role in detonation initiation has been recognized for many years. Flow visualization data show that the flame front gets thicker suddenly, and then the onset of detonation develops. In most of literatures, the flame acceleration was taken as the whole DDT process, in which many physical issues were included, even the hotspot explosion. In this paper, the reaction zone acceleration will be investigated and defined as one of the elementary phenomena for detonation initiation. It is expected that the thus-defined reaction zone acceleration can serve as a primary phenomenon like the hotspot for describing detonation initiation and propagation. 

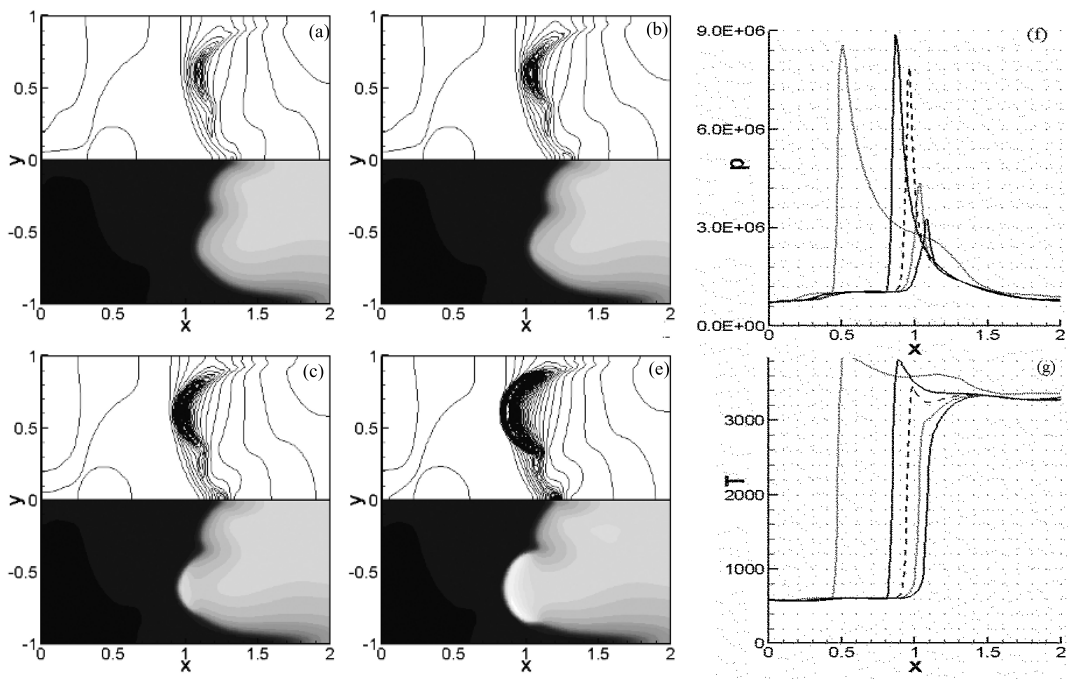

Fig. 4. Sequential pressure (upper) and temperature (lower) distributions during the reaction zone acceleration with sequential pressure and temperature profiles across the reaction zone

Detonation initiation developed from Richtmyer-Meshkov instability of the reaction zone in the second case is presented in Fig.4, and the upstream-travelling reaction zone is shown with pressure distributions in the upper half and temperature variations in the lower half of each picture. A series of spontaneous waves resulting from the disturbed reaction zone are also observable in Fig.4(a). These compression waves are expected to propagate in all directions, but their speeds are affected dramatically by the local thermo-dynamic environment. The downstream-propagating waves leave the reaction zone successively since products temperature behind the reaction zone is almost uniform. The upstream-propagating waves pile up in the front of the reaction zone because of the sudden temperature change from products to reactants. The reaction zone and its temperature front, as shown in Fig.4(a), are convex, so, the post-shock pressure and temperature should fall because of the front diverging effect. However, the isobars in Fig.4(b) becomes denser than those in Fig.4(a), which indicates the more spontaneous waves are generated in the reaction zone and accumulated in its front. Although the convex surface divergence is likely to make the post-shock pressure and temperature fall, the more and more intensive reactions generate the stronger and stronger compressive waves which not only compensate the front diverging effect, but also increase temperature further. The temperature peak forms at the tip of the convex reaction front, and meanwhile the corresponding pressure also becomes higher, as shown in Fig.4(c) where the isobars get more denser. And then, a arc detonation wavelet forms in Fig.4(d) and engulfs the flame front below it. Finally a cellular detonation develops. Detonation initiation occurs on the convex reaction front, and the coupling of pressure waves with successively increasing reactions are fundamentally important.

Time sequence of pressure and temperature profiles along the line of $y=0.6$ during reaction zone acceleration are plotted in Fig.4(e) and (f). The profiles can be ordered from right to left according to their successive time instants. The line of $y=0.6$ passes 
exactly the middle of the convex reaction zone, the parameters along which are useful to demonstrate the reaction zone acceleration. The first pressure profile shows a pressure pulse which indicates the beginning of spontaneous wave accumulation. The peak pressure is getting higher and higher as the reaction zone propagates upstream, and the corresponding temperature front is getting steeper and steeper. This phenomenon indicates the leading shock generation and reaction rate increasing. The peak pressure reaches to its maximum at the fifth profile as shown in Fig.4(e) and the value is about 9.0 MPa. The peak pressure decreases slightly at later instants and remains almost constant since then. Meanwhile a little pulse occurs in the fourth temperature profile and the temperature peak reaches about $3800 \mathrm{~K}$ in the fifth profile, as shown in Fig.4(f). The maximum temperature finally reaches to $4000 \mathrm{~K}$ and maintains almost constant since then, and a detonation forms. It is observed that the overshot of the post-shock pressure is slight and the post-shock temperature reaches to the C-J value gradually.

The positive feedback loop is also underlying the hotspot-induced DDT, but there are two primary physical issues that tell differences between the hotspot-induced DDT and the reaction zone acceleration. One of them is that the hotspot explosion happens in an unburned gas pocket, more intensive heat release is induced, and an over-driven detonation develops. For the reaction zone acceleration, chemical reactions take place only in a narrow zone, and no obviously over-driven phenomenon is observed. The other issue is that external perturbations are in need to trigger the hotspot explosion when the unburned gas pocket reaches to a critical thermo-state, otherwise the hotspot may die out without such perturbations.

Being similar to detonation initiation, cellular detonation propagation also depends on these mechanisms. Hotspots are generated from triple-point collisions, and the reaction zone follows incident shocks. The complex of the incident shock and reaction zone is alternately accelerating and decelerating around the C-J state. The acceleration is supported by the hotspot explosion and the deceleration results from the convex front diverging. Their interaction makes the cellular detonation be self-sustained, and there would not be any practical detonation if without either of them. Therefore, the detonation propagation and initiation go on the same primary mechanisms.

\section{Conclusion}

The hotspot and the reaction zone acceleration are two primary physical mechanisms underlying the deflagration to detonation transition. The first mechanism may be dominate in some cases and the second in other cases, but in most cases, they are observed together. The two mechanisms are also essential issues for cellular detonations, their interaction supports the steady propagation of practical detonations.

\section{References}

1. John H. S. Lee, Initiation of Gaseous Detonation, Ann. Rev. Phys. Chem, 28:75-104, 1977

2. John. H. S. Lee and A. J. Higgins, Comments on Criteria for Direct Initiation of Detonation. Phil. Trans. R. Soc. Lond. A, 357: 3503-3521, 1999

3. Elaine S. Oran, Vadim N. Gamezo: Origins of the Deflagration to detonation Transition in Gas-phase Combustion. Combust. Flame 2006.

4. A. K.Oppenheim, R. I. Soloukhirt, Experiments in Gas Dynamics of Explosions. Ann. Rev. Fluid Mech., 5:31-58, 1973. 